\title{
Supporting Adaptation of Wireless Communication Protocols
}

\author{
LACHHMAN DAS DHOMEJA*, IRFAN ALI SOOMRO*, AND YASIR ARFAT MALKANI** \\ RECEIVED ON 23.02.2015 ACCEPTED ON 14.12.2015
}

\begin{abstract}
Pervasive devices such as mobile phones and PDAs (Personal Digital Assistants) come with different wireless communication capabilities, for example, WiFi (Wireless Fidelity), Bluetooth, IrDA (Infrared), etc. In order for pervasive devices to interact with each other, they need to have matching (alike) communication capabilities, otherwise such heterogeneous devices would not be able to interact with each other. In this paper we address this issue and propose a system that makes devices with heterogeneous wireless communication capabilities communicate with each other. The proposed system supports adaptation of wireless communication protocols through a proxy, which sits between a client and a server, and supports adaptation of wireless communication protocols. Its functionality involves intercepting a request made by a client with a different wireless communication capability (e.g. Bluetooth) from what the server has (e.g. WiFi), connecting to the server and then sending results back to the client. We have tested the system by implementing a messaging service application and running it on the system. The proxy supports all Bluetooth protocols, i.e. OBEX (Object Exchange), L2CAP(Logical Link Control and Adaptation Protocol), RFCOM (Radio Frequency Communication) and WiFi protocol and can run on (J2MW (Java 2 Micro Edition) enabled mobile phones which support both Bluetooth and WiFi capabilities.
\end{abstract}

Key Words: Wireless Communication Protocols, Adaptation, WiFi, Bluetooth, J2ME.

\section{INTRODUCTION}

$\mathrm{T}$ he availability of computing power within small mobile devices along with the wireless communication capabilities has made them an integral part of our everyday life. Because of this, the use of mobile phones is not restricted to making calls only, but also enables us to access variety of applications, such as text messaging, internet services and thus allowing accessing information of our interest anytime anywhere. The mobile devices are equipped with different wireless communication capabilities, for example,
Bluetooth, WiFi, IrDA and each wireless capability has different communication characteristics. Bluetooth is a short range wireless communication technology, which is used for exchanging the data over short distance. Bluetooth technology connects nearby devices at the data rate of 1-3 Mbps and automatically searches for devices within its radio range (15-30m) [2].

WiFi provides wireless access to applications and data across a radio network and it sets up numerous ways to

* Institute of Information \&Communication Technology, University of Sindh, Jamshoro.

** Institute of Mathematics \& Computer Science, University of Sindh, Jamshoro.

Mehran University Research Journal of Engineering \& Technology, Volume 35, No. 4, October, 2016 [p-ISSN: 0254-7821, e-ISSN: 2413-7219] 
build up a connection between the transmitter and the receiver, such as DSSS (Direct-Sequence Spread Spectrum), FHSS (Frequency Hopping Spread Spectrum) and OFDM (Orthogonal Frequencies Division Multiplexing) [1,2]. Accessing information through WiFi is far quicker than the conventional modem over a large network and allows users to connect to internet from any place such as their home, office or a public place without wires.

Wireless devices are able to access information anytime anywhere using these wireless communication technologies. There exists a large body of research focusing on mobile computing which addresses various issues of mobile computing. For example, the large body of research in mobile environments focuses on handling the inherent limitations of mobile technology, such as small CPU power, variable bandwidth connectivity, small screen size, etc [3-12]. Another aspect of mobile environment which has been addressed in the literature is wireless communication protocols adaptation, which allows mobile devices to continue accessing services using different wireless communication protocols while on move. For example, if a device is currently accessing service using blue-tooth wireless system and WiFi connectivity has become available, the system would automatically select the WiFi wireless system and allow device to continue accessing multimedia service using WiFi connectivity. For a mobile device to be able to continue accessing service using different wireless communication systems is required to be equipped with the support of all network technologies (e.g. WiFi, UMTS, and Bluetooth). But what if a device does not have support for these underlying network technologies”. We address this issue and put forward a proxy-based system which provides switching between heterogeneous wireless communication protocols. Our research idea is based on the fact that two communicating devices may not have the same wireless communication capabilities (e.g. WiFi, Bluetooth, etc.), but are still able to communicate with each other. As a proof of concept, we have designed and implemented the system which provides wireless communication protocol adaptation support, which enables small devices with dissimilar wireless communication protocols communicate with each other.

The remaining part of the paper is organized as follows: Section 2 provides the related work. Section 3 provides the high-level description of proposed approach. Implementation and discussion of the proposed approach is presented in section 4. Conclusion is given in section 5 and future work is presented in section 6 .

\section{RELATED WORK}

Akyildiz, et. al. [13] have developed the NGWS (Next Generation Wireless System), which integrates different wireless technologies (i.e. Bluetooth, WiFi, GPS, 3G Cellular Network). This allows mobile users to access highspeed data (i.e. multimedia services) anywhere and anytime using these different wireless systems. The functionality of their system involves selecting a best network based on user's service needs. For example, if a device is currently accessing a multimedia service using the Bluetooth wireless system and WiFi connectivity has become available, the system would automatically select the WiFi and allow the device to continue accessing multimedia service. To summarize, their system provides adaptation support in terms of switching between various wireless systems based on user's service needs. We also focus on providing adaptation of communication protocol switching but when two devices communicating with each other have different wireless communication protocols.

Vegni [14] argue that the heterogeneous networks for next generation systems can operate together to support seamless mobility, allowing users to remain connected with the services (using heterogeneous networks) while on the move. Mobility support is a form of adaptation in which network connectivity is adapted, i.e. from one network connectivity (e.g. UMTS) to other network

Mehran University Research Journal of Engineering \& Technology, Volume 35, No. 4, October, 2016 [p-ISSN: 0254-7821, e-ISSN: 2413-7219] 
connectivity (e.g. WLAN) based on some conditions. The device being used by the user for accessing services is required to be equipped with the support of all network technologies (e.g. WiFi, UMTS, Bluetooth) to be able to remain connected with the service through these technologies. However, if the device does not have support for these underlying network technologies, it will not be able to take advantage of adaptation support (i.e. which provides supports for switching between these network technologies). As a result, the user cannot maintain connectivity with the service while on the move, affecting user experience. We also provide adaptation support which provides switching between heterogeneous wireless communications but with a different focus. Our research idea is based on the fact that two communicating devices may not have same wireless communication capabilities (e.g. WiFi, Bluetooth, etc.), but are still able to communicate with each other.

Lufei and Shi [15] have developed an application level adaptation framework, called Fractal based on mobile code technology for protocol adaptation, which uses existing CDN (Content Distribution Networks) for protocol adaptors (mobile codes) for deployment. The proposed framework is based on the idea that there may be heterogeneous devices in the environment and the application server can communicate to these devices through different application protocols. At runtime a suitable application protocol is chosen and then communication between client device and the application server takes place using that protocol. However, we focus on the adaptation of communication protocols in which two devices with different communication protocols (e.g. Bluetooth, WiFi, etc.) are able to communicate with each other.

Rosa, et. al. [16] focus on supporting adaptation of protocol stack. This work is based on the idea that each protocol offers a specific quality of service and the performance of the application can be improved by using a different protocol tailored to the current conditions.
The protocol stack is a composition of different protocols and each is suitable for particular conditions. The protocol stack adaptation process involves switching from one protocol to another in reaction to changes in the environment, offering the best possible quality of service. Another contribution of their research is a proposition of policy-based approach in which adaptation logic is expressed in a high-level policy. The advantage of policybased approach is decoupling between adaptation logic and protocol logic, thus reducing development effort.

Etemadi, et. al. [17] have developed a platform which allows controlling wearable devices (for example, those used in medical and fitness applications) from the web using a smart phone as a smart proxy. The smart proxy is acting as a bridge/router between BLE (Bluetooth Low Energy) and Internet - it connects BLE devices with their cloud services through internet (e.g. via WiFi). The functionality of smart proxy involves performing switching between WiFi and Bluetooth protocol. In contrast to this, the proxy in our proposed system has capability of connecting BLE client to WiFi server and WiFi client to BLE server.

\section{THE PROPOSED APPROACH}

The proposed system supports adaptation of wireless communication protocols, which enables two dissimilar devices (with different communication capabilities) to communicate with each other. The main component of the proposed system is a proxy server that makes this happens. Ideally the system should be capable of performing switching between various communication protocols; however, the current implementation of the proposed system provides switching between two communication protocols only, i.e. Bluetooth to WiFi and vice versa.

The high-level architecture of the proposed system is shown in Fig. 1. The system architecture has three components: Client, Proxy and Server. Each of these components is described in subsequent subsections.

Mehran University Research Journal of Engineering \& Technology, Volume 35, No. 4, October, 2016 [p-ISSN: 0254-7821, e-ISSN: 2413-7219] 


\subsection{Client}

The client in general requests the server for some services or resources. In the proposed system, the client may be Bluetooth-enabled or WiFi-enabled. The Bluetooth client gets connected to the WiFi server through the proxy server to be able to access the services offered by the $\mathrm{WiFi}$ server. The sequence diagram for connectivity between Bluetooth client and WiFi server via the proxy is shown in Fig. 2.
Similarly, the Wifi client gets connected to the Bluetooth server through the proxy server to be able to access services offered by the Bluetooth server. The message sequence diagram illustrating the connectivity between WiFi client and Bluetooth server via the proxy is shown in Fig. 3.

\subsection{Proxy}

The key to system design is the proxy component, which is responsible for providing protocol adaptation support.

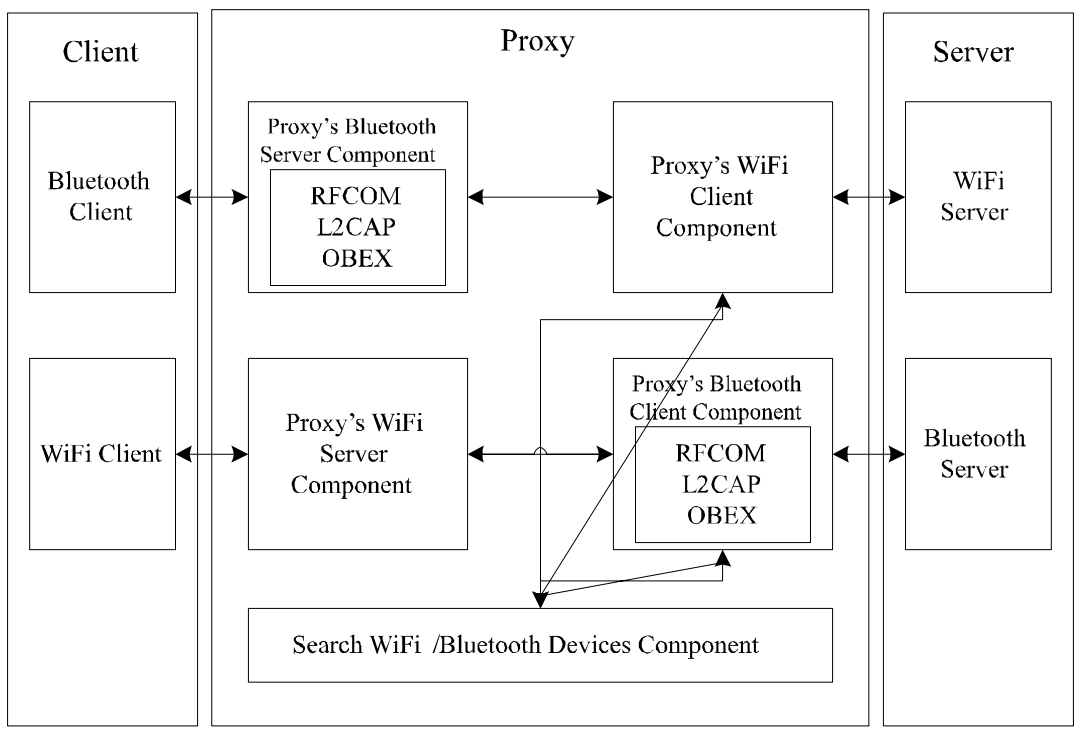

FIG. 1. HIGH-LEVEL ARCHITECTURE OF THE PROPOSED SYSTEM

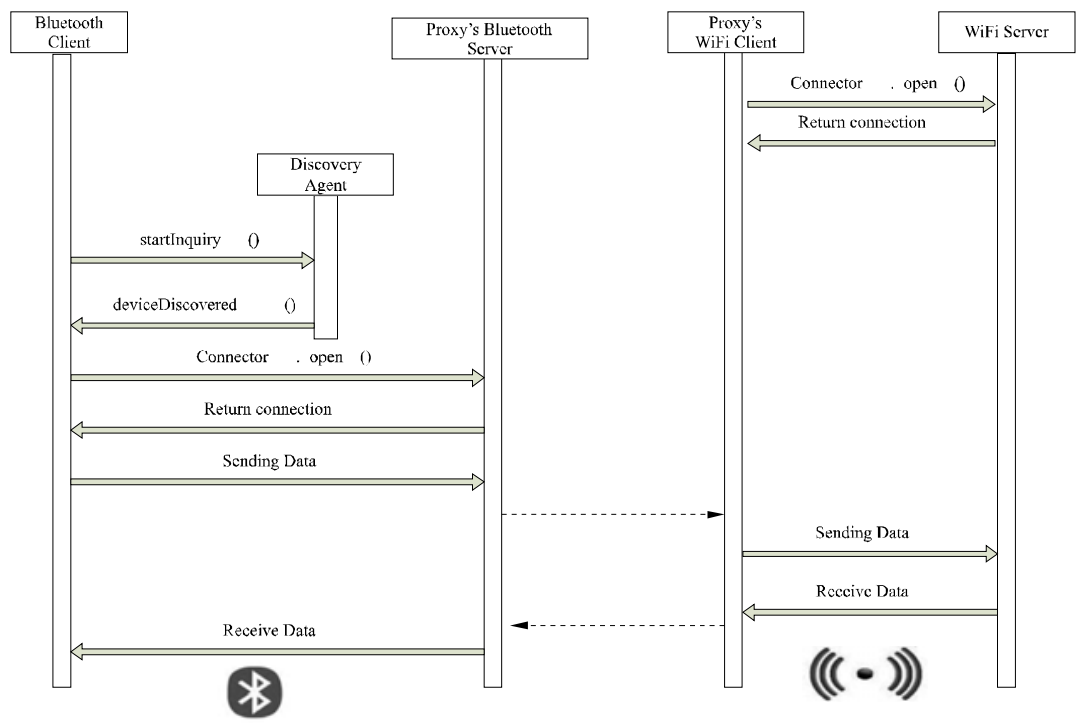

FIG. 2. INTERACTIONS BETWEEN BLUETOOTH CLIENT TO WIFI SERVER THROUGH THE PROXY

Mehran University Research Journal of Engineering \& Technology, Volume 35, No. 4, October, 2016 [p-ISSN: 0254-7821, e-ISSN: 2413-7219] 
This component allows client and server with dissimilar communication capabilities to interact with each other. For example, the device with Bluetooth communication capability wants to communicate with another device, which does not have the same communication capability (i.e., Bluetooth), but has a different communication capability (e.g. WiFi). The proxy sitting in between intercepts Bluetooth-enabled device's request, makes a connection with WiFi enabled device and communicates with it on behalf of Bluetooth-enabled device, and sends the results back to Bluetooth-enabled device.

\subsection{Server}

The server is a software component that waits for clients to connect to it and provides the services requested by the client. The proposed system supports connectivity between (1) Bluetooth client and Wifi Server and (2) WiFi client to WiFi server.

\section{IMPLEMENTATIONAND DISCUSSION}

The system implementation involves the development of proxy server for establishing communication between client (i.e. Bluetooth client or WiFi client) and server (i.e. Bluetooth server or WiFi server). The system has been tested by developing and running a simple Messaging application on Bluetooth enabled mobile device and WiFi device.

Fig. 4 shows a screenshot of the proxy server after it has been started on a mobile device. The search devices option enables the proxy to search all the compatible Bluetooth or WiFi devices within the range.

Before the proxy is run, it is to be ensured that the Bluetooth-enabled and WiFi-enabled servers are active so that proxy is able to search them. Fig. 5 shows the interface of proxy server searching for any Bluetooth and WiFi devices.

The proxy server displays information of all connected Bluetooth or WiFi devices as shown in Fig. 6. If no device is active then the search result displays no device found message. Client application gets connected with the server (i.e. Bluetooth server, WiFi server) through the proxy.

Once devices are connected, the user can proceed to an input screen to enter some text message, which will be sent to the WiFi server (running messaging application) as shown in Figs. 7-8.

Figs. 9-10 show the message exchange between $\mathrm{WiFi}$ client and Bluetooth server.

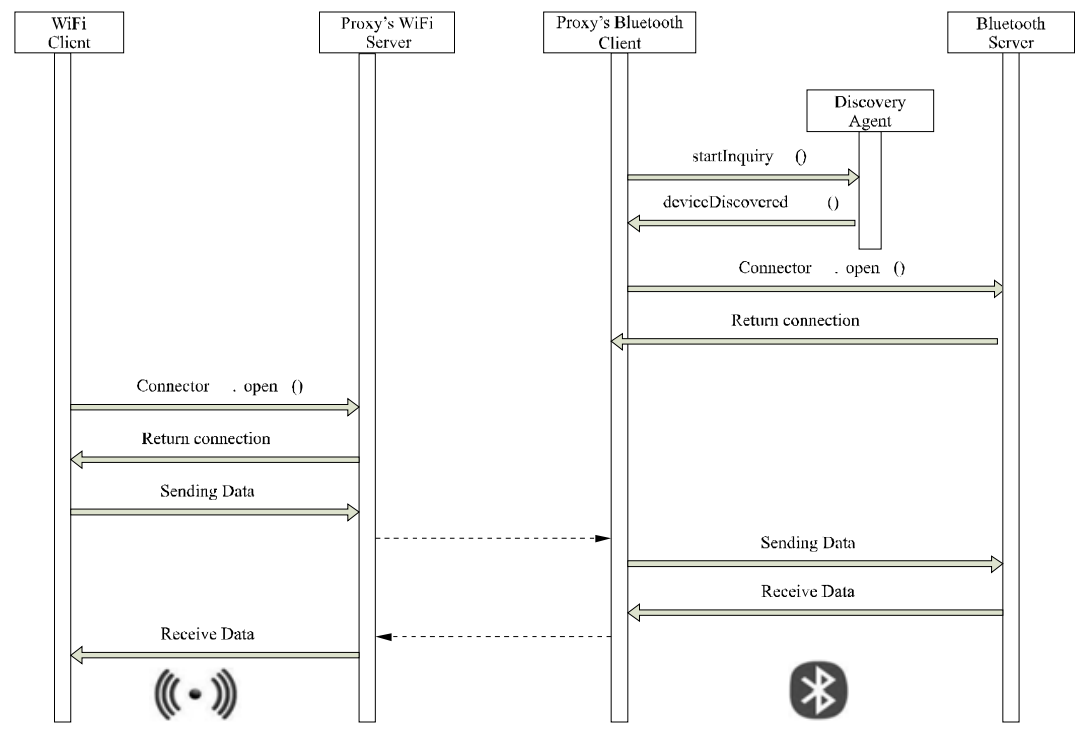

FIG. 3. INTERACTIONS BETWEEN WIFI CLIENT AND BLUETOOTH SERVER THROUGH THE PROXY

Mehran University Research Journal of Engineering \& Technology, Volume 35, No. 4, October, 2016 [p-ISSN: 0254-7821, e-ISSN: 2413-7219] 


\subsection{Discussion}

The main component of the implemented system is a proxy server, which is capable of supporting different wireless communication protocols (e.g. Bluetooth, WiFi, etc). The main functionality of this component is the support for adaptation of wireless communication protocols in which

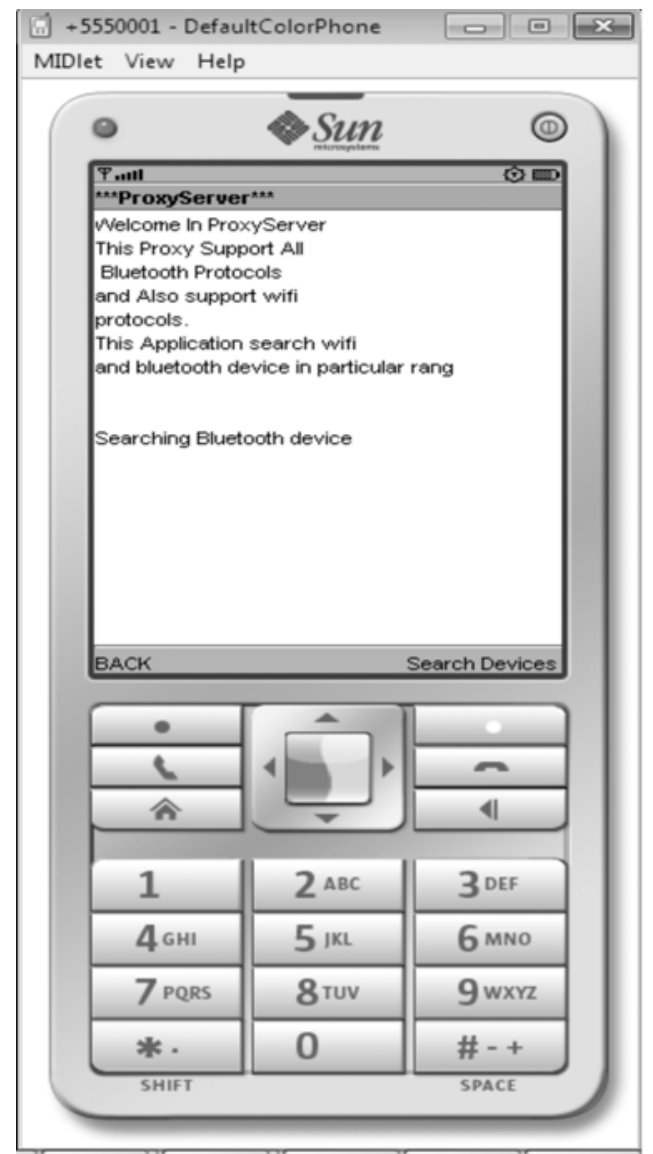

FIG. 4. THE SNAPSHOT OF PROXY SERVER RUN IN A MOBILE DEVICE

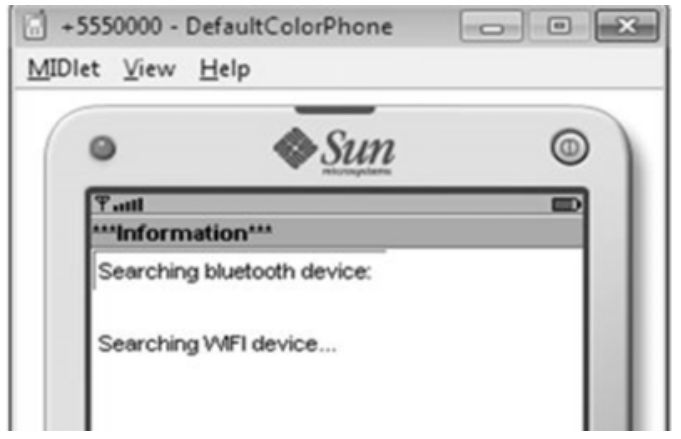

FIG. 5. THE PROXY SERVER SEARCHING FOR BLUETOOTH AND WIFI DEVICES adaptation support of proxy makes two devices with different communication capabilities (e.g. one with Bluetooth capability and other with WiFi capability) communicate with each other. The proxy server supports all Bluetooth protocols (i.e. OBEX, L2CAP, RFCOM) and WiFi protocols and can run on J2ME enabled mobile phones which support both Bluetooth and WiFi capabilities. It also has capabilities to search devices such as WiFi devices or Bluetooth devices in limited range. If WiFi or Bluetooth device is found by the Proxy server, then it will get permission from user for establishing connection with particular searched devices.

In some scenarios, it might be the case that there are multiple devices with the same wireless communication capability and offering a particular service which is required by the device with a different wireless communication capability. For example, Bluetoothenabled device wants to access the printing service, which is being offered by more than one WiFi enabled device or vice versa. This is one area where the functionality of the proxy can be extended to address this issue in future.

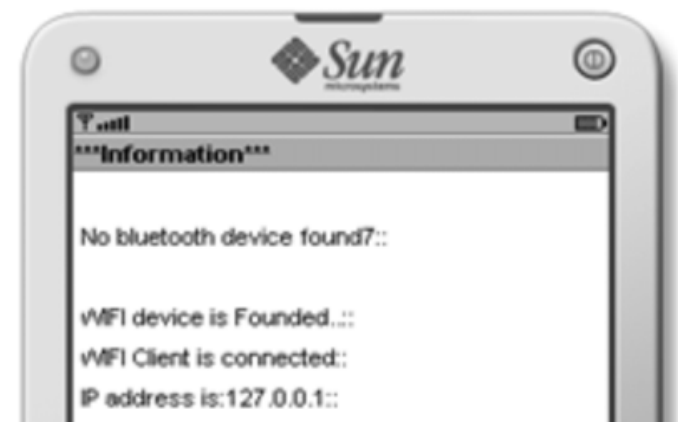

FIG. 6. A LIST OF ALL COMPATIBLE DEVICES FOUND BY THE PROXY SERVER.

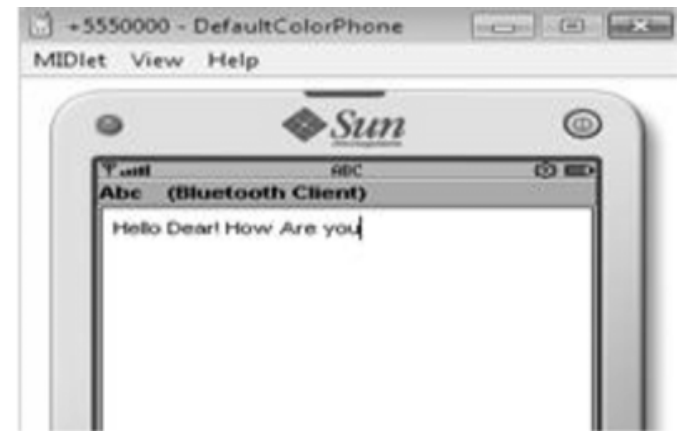

FIG. 7. SENDING TEXT MESSAGE FROM A MOBILE DEVICE

Mehran University Research Journal of Engineering \& Technology, Volume 35, No. 4, October, 2016 [p-ISSN: 0254-7821, e-ISSN: 2413-7219] 


\section{CONCLUSION}

In this paper, we have addressed the research question of what if two pervasive devices want to interact with each other, but having different wireless communication protocols. As a proof of concept, we have designed and implemented the system that enables small devices with dissimilar wireless communication capabilities to communicate with each other. We have tested the system by implementing a messaging service application and running it on the system. One device was Bluetoothenabled and other device was WiFi-enabled and both were able to send and receive messages to/from each other.

\section{FUTURE WORK}

The work presented in the paper can further be extended in two directions:

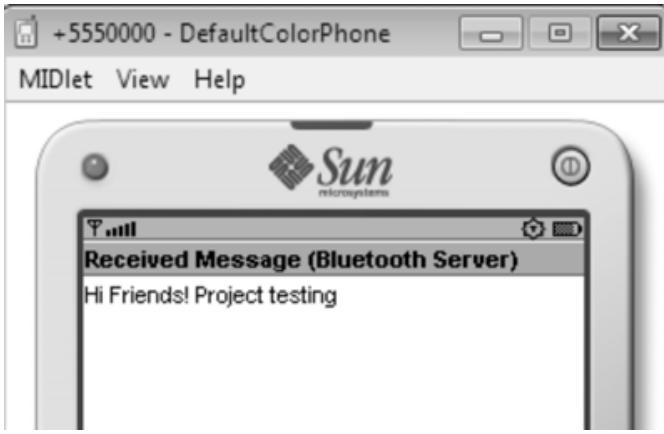

FIG. 10. MESSAGE RECEIVED ON A BLUETOOTH ENABLED DEVICE

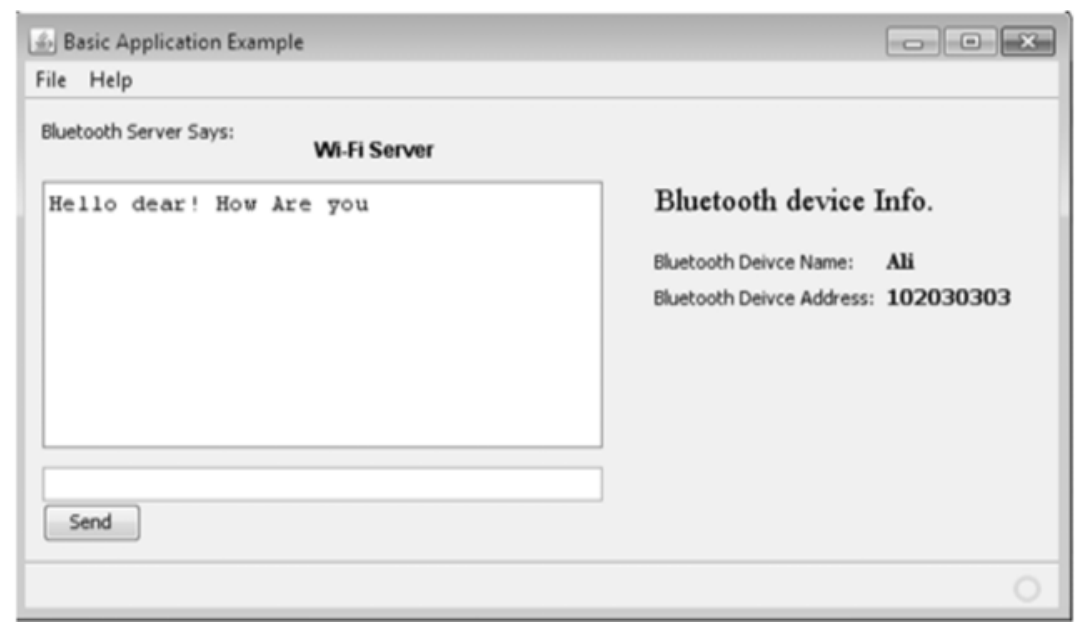

FIG. 8. RECEIVING TEXT MESSAGE ON A WIFI DEVICE

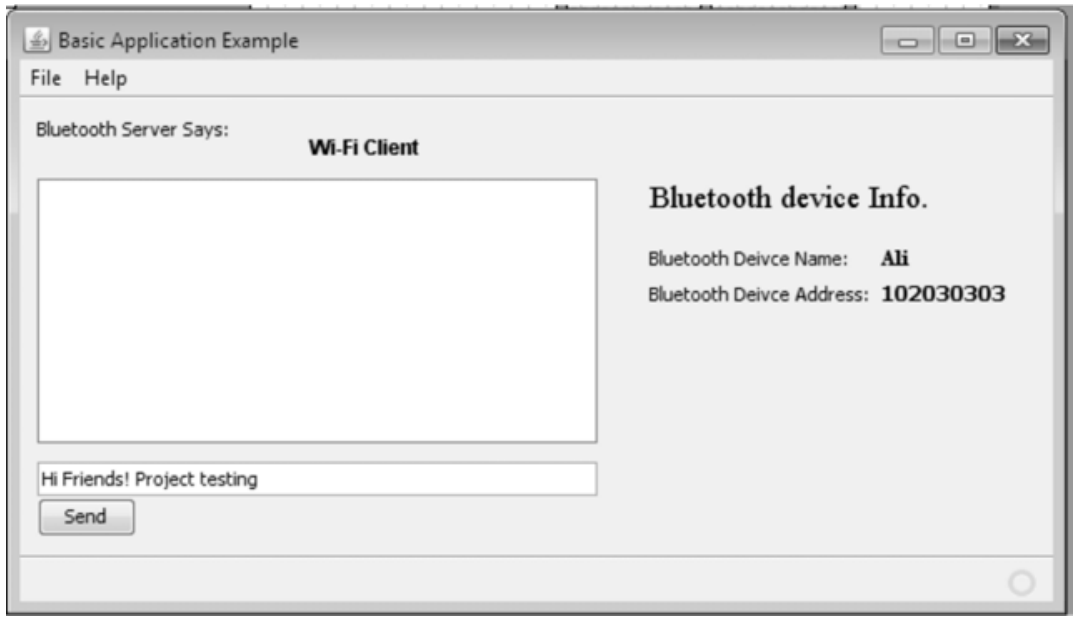

FIG. 9. SENDING MESSAGE FROM A WIFI ENABLED DEVICE

Mehran University Research Journal of Engineering \& Technology, Volume 35, No. 4, October, 2016 [p-ISSN: 0254-7821, e-ISSN: 2413-7219] 
(i) The proxy has been implemented for J2ME devices only, which means the system cannot be ported across other platforms, such as Android-based and IOS-enabled devices. The portability can be achieved by providing the cross-platform implementation of the proposed system.

(ii) Currently, the proxy enables Bluetooth-enabled devices to communicate with WiFi enabled devices and vice versa for text-based messages only. The functionality of the proxy can further be extended to support other types of applications, such as FTP, headset, voice communication, printing and video communication.

\section{ACKNOWLEDGEMENT}

This work has been carried out jointly by the authors in the Institute of Information \& Communication Technology, University of Sindh, Jamshoro, Pakistan.

\section{REFERENCES}

Mishra, A.R., "Cellular Technology in Emerging Markets”, Cellular Technologies for Emerging Markets: 2G, 3G and Beyond”, pp. 326, [ISBN: 0470779470], July, 2010.

Labiod, H., Hossam, A., and Costantino, D.S., ”WIFITM, Bluetooth TM, Zigbee TM and WiMAXTM", Springer Science \& Business Media, 2007.

Bellavista, P., Corradi, A., Montanari, R., and Stefanelli, C., "Context-Aware Middleware for Resource Management in the Wireless Internet", IEEE Transactions on Software Engineering, Volume 29, No. 12, pp. 1086-1099. 2003.

] David, P.C., and Ledoux, T., "Towards a Framework for Self-Adaptive Component-Based Applications", Distributed Applications and Interoperable Systems, pp. 1-14, Springer Berlin Heidelberg. 2003.

[5] Keeney, J., and Cahill, V., "Chisel: A Policy-Driven, Context-Aware, Dynamic Adaptation Framework", Proceedings of IEEE 4th International Workshop on Policy, pp. 3-14, 2003.
Sousa, J.P., and Garlan, D., “Aura: An Architectural Framework for User Mobility in Ubiquitous Computing Environments”, Software Architecture, pp. 29-43. Springer, USA, 2002.

[7] Floch, J., Hallsteinsen, S., Stav, E., Eliassen, F., Lund, K., and Gjorven, E., "Using Architecture Models for Runtime Adaptability”, IEEE Software, Volume 23, No. 2, pp. 62-70, 2006.

[8] Noble, B., "System Support for Mobile, Adaptive Applications”, IEEE Personal Communications, Volume 7, No. 1, pp. 44-49. 2000.

[9] Noble, B.D., and Satyanarayanan, M., "Experience with Adaptive Mobile Applications in Odyssey", Mobile Networks and Applications, Volume 4, No. 4, pp. 245-254. 1999.

[10] Davies, N., Finney, J., Friday, A., and Scott, A., "Supporting Adaptive Video Applications in Mobile Environments”, IEEE Communications Magazine, Volume 36, No. 6, pp. 138-143. 1998.

[11] Fox, A., Gribble, S.D., Chawathe, Y., and Brewer, E.A., "Adapting to Network and Client Variation Using Infrastructural Proxies: Lessons and Perspectives”, IEEE Personal Communications, Volume 5, No. 4, pp. 10-19, 1998.

[12] Fox, A., Gribble, S.D., Brewer, E.A., and Amir, E., "Adapting to Network and Client Variability via OnDemand Dynamic Distillation”, ACM SIGOPS Operating Systems Review, Volume 30, No. 5, pp. 160-170. 1996.

[13] Akyildiz, I.F., Mohanty, S., and Xie, J., “A Ubiquitous Mobile Communication Architecture for NextGeneration Heterogeneous Wireless Systems”, IEEE Communications Magazine, Volume 43, No. 6, pp. S29-S36. 2005.

[14] Vegni, A.M., "Multimedia Mobile Communications in Heterogeneous Wireless Networks - Part-2”, Ph.D. Thesis, University of Roma Tre, March 2010, (Available Online at: http://www.comlab.uniroma3.it/vegni.htm)

[15] Lufei, H., and Shi, W., "Fractal: A Mobile Code Based Framework for Dynamic Application Protocol Adaptation in Pervasive Computing”, Proceedings of $19^{\text {th }}$ IEEE International Symposium on Parallel and Distributed Processing, 2005.

[16] Rosa, L., Lopes, A., and Rodrigues, L., "Policy-Driven Adaptation of Protocol Stacks", IEEE International Conference on Autonomic and Autonomous Systems, 2006.

[17] Etemadi, M., McGrath, W., Shuvo, R., and Bjoern, H., "Fabryq: Using Phones as Smart Proxies to Control Wearable Devices from the Web”, Technical Report No. ULB/EECS, Department of Electrical Engineering \& Computer Science, California University, Berkeley, 2014. 